\title{
Generation of internal gravity waves by an oscillating horizontal disc
}

\author{
By P. A. Martin ${ }^{1, *}$ and Stefan G. Llewellyn Smith ${ }^{2}$ \\ ${ }^{1}$ Department of Mathematical and Computer Sciences, Colorado School of \\ Mines, Golden, CO 80401-1887, USA \\ ${ }^{2}$ Department of Mechanical and Aerospace Engineering, Jacobs School of \\ Engineering, UCSD, 9500 Gilman Drive, La Jolla, CA 92093-0411, USA
}

Internal gravity waves are generated in a stratified fluid by arbitrary forced oscillations of a horizontal disc. The wave fields are calculated in both the time domain and the frequency domain. In the time domain, an initial-value problem is solved using Laplace transforms; causality is imposed. In the frequency domain (time-harmonic oscillations), a radiation condition is imposed: a plane-wave (Fourier) decomposition is used in which waves with outgoing group velocity are selected. It is shown that both approaches lead to the same solution, once transient effects are ignored. Then, a method is given for calculating the far field, using asymptotic approximations of double integrals. It is shown that the total energy flux is outwards, for arbitrary forcings of the disc. Further investigations of energy transport are made with a view to clarifying the nature of radiation conditions in the frequency domain.

Key words: Internal gravity waves; dual integral equations; oscillating disc.

\section{Introduction}

It is well known that internal gravity waves exist in a density stratified fluid, and that they can be generated by oscillating immersed bodies or by scattering from topography. The motivating problem here is to calculate these wave fields, in a three-dimensional unbounded fluid domain. It is also known that the energy produced by the oscillations is confined to characteristic conical wave beams. See, for example, Lighthill, 1978, chapter 4, or LeBlond \& Mysak, 1978, §8.

Under the Boussinesq approximation, with an inviscid fluid, a uniform density stratification and small-amplitude motions, we can write down the governing equations (see $\S 2$ for details). In particular, for time-harmonic motions, the pressure is $\operatorname{Re}\left\{p \mathrm{e}^{-\mathrm{i} \omega t}\right\}$, where $p(x, y, z)$ solves

$$
\frac{\partial^{2} p}{\partial x^{2}}+\frac{\partial^{2} p}{\partial y^{2}}-\frac{\omega^{2}}{N^{2}-\omega^{2}} \frac{\partial^{2} p}{\partial z^{2}}=0
$$

$z$ is the vertical coordinate and $N$ is the constant Brunt-Väisälä frequency. We are interested in the case where $0<\omega<N$ so that (1.1) is hyperbolic. This equation

Author for correspondence (pamartin@mines.edu)

Proc. R. Soc. A 1-17; doi: $10.1098 /$ rspa. 00000000

This journal is (c) 2011 The Royal Society 
has to be solved subject to a boundary condition (prescribed normal velocity) and a far-field (radiation) condition.

There are several ways to specify the radiation condition in the frequency domain; for a review, see Voisin $(1991, \S 3.2)$. One way is to replace $\omega$ by $\omega+\mathrm{i} \varepsilon$, letting $\varepsilon \rightarrow 0$ at the end of the calculation (Lighthill, 1978, §4.9). Another way uses analytic continuation in the complex $\omega$-plane; see below for more details. When plane-wave decompositions are available, we can examine the corresponding group velocity; note that, for plane internal waves, group and phase velocities are orthogonal (Lighthill, 1978, §4.4). Barcilon \& Bleistein (1969) used a local planewave approximation at each point in the fluid but their approach was criticised by Baines (1971).

Underlying radiation conditions in the frequency domain is the requirement of causality in the time domain. Therefore, because of the variety of frequencydomain radiation conditions, we start by treating an initial-value problem. The problem we have chosen concerns an oscillating horizontal disc: there is no motion for $t<0$, and then time-harmonic forcing is switched on at $t=0$. We solve the resulting problem, using Laplace transforms in time and Fourier transforms in the horizontal coordinates $x$ and $y$. This leads to solvable dual integral equations. Inverting the Laplace transform, imposing causality, gives a transient contribution plus a time-harmonic forced response; we are interested in the latter.

Next, we formulate the analogous time-harmonic problem. A radiation condition is imposed in the Fourier domain: we ensure that only those plane-wave contributions with group velocity directed away from the disc are retained. This approach has been used by others: it is limited to those problems where planewave decompositions are available throughout the fluid domain. We find that the pressure field obtained is exactly the same as the forced response obtained in the initial-value problem. (This is an example of the so-called Limiting Amplitude Principle.)

Having confirmed that our time-harmonic solution does respect causality, we go on to estimate far-field quantities $(\S 7)$. This requires the asymptotic estimation of certain double integrals; a form of the method of stationary phase is used, in which the stationary-phase points occur along a line in the domain of integration. Estimates for both the pressure, $p$, and the velocity, $\boldsymbol{v}$, within the wave beams are obtained. Then, the time-averaged energy transport vector, I, defined by

$$
\mathbf{I}=\left(\rho_{0} / 2\right) \operatorname{Re}\{p \overline{\boldsymbol{v}}\},
$$

can be calculated, where the overbar denotes complex conjugation and $\rho_{0}$ is the constant background density. The equations of motion imply that $\operatorname{div} \mathbf{I}=0$. Consequently, if $S$ is a closed surface enclosing the oscillating plate, then we should have $\int_{S} \mathbf{I} \cdot \boldsymbol{n} \mathrm{d} S>0$, where $\boldsymbol{n}$ is the unit normal on $S$ pointing away from the plate. We verify this (global) inequality in the far field, for arbitrary forcings of the plate $(\S 7 \mathrm{c})$.

It is clear that $\mathbf{I}$ is arbitrary in the sense that it could be replaced by $\mathbf{I}+\mathbf{I}_{1}$ provided that $\operatorname{div} \mathbf{I}_{1}=0$ (Longuet-Higgins 1964). This means that there is no reliable physical interpretation of $\mathbf{I} \cdot \boldsymbol{n}$, although Lighthill $(1978$, p. 14) states that it gives the energy 'being transported in the direction of $\boldsymbol{n}$ across a small plane element at right angles to $\boldsymbol{n}$ '. Nevertheless, we might expect that $\mathbf{I} \cdot \boldsymbol{n}>0$, pointwise. However, we show, by example, that this is not the case. This confirms 
that examining the sign of $\mathbf{I} \cdot \boldsymbol{n}$ at each point in the far-field cannot be used as a radiation condition: doing so could exclude physically meaningful solutions.

Let us conclude this introduction by mentioning previous work on the generation of three-dimensional internal gravity waves by oscillating bodies. Most of it is concerned with axisymmetric motions of spheres and spheroids; see, for example, Hendershott (1969), Sarma \& Krishna (1972), Lai \& Lee (1981), Appleby \& Crighton (1987), Voisin (1991, 2003), Flynn et al. (2003) and Voisin et al. (2010). The approach of these authors starts by solving (1.1) when $\omega>N$ using a scaling in $z$. This leads to a boundary value problem for Laplace's equation exterior to a spheroid, a problem that can be solved by separation of variables. The solution for $\omega<N$ is then obtained by analytic continuation. In particular, Sarma \& Krishna (1972) and Lai \& Lee (1981) obtained the solution for vertical oscillations of a rigid disc; we find agreement with their solutions ( $\S 6 \mathrm{a})$.

For disc problems, there is a paper by Gabov \& Pletner (1988), in which the pressure (not the normal velocity) is prescribed on the disc. There is the recent paper by Davis \& Llewellyn Smith (2010) where the plate oscillates in its own plane but the fluid is viscous. There are also several papers by Chashechkin and his collaborators, in which internal waves are generated in a viscous fluid by a circular piston in a horizontal rigid plane, $z=0$. Mathematically, this problem is simpler than a disc problem because the velocity is prescribed over the whole plane, $z=0$. See, for example, Chashechkin et al. (2004) and Bardakov et al. (2007); these papers include both theoretical and experimental results.

\section{Mathematical formulation}

We consider a variable density fluid without rotation. We use the linearized form of the Boussinesq equations (Vallis, 2006, §2.4.2),

$$
\partial \tilde{\boldsymbol{v}} / \partial t=-\operatorname{grad} \tilde{p}+\tilde{b} \hat{\mathbf{z}}, \quad \operatorname{div} \tilde{\boldsymbol{v}}=0, \quad \partial \tilde{b} / \partial t+N^{2} \tilde{w}=0 .
$$

Here, we have Cartesian coordinates $O x y z$, with $z$ pointing upwards; $\hat{\mathbf{z}}$ is a unit vector in the $z$-direction. The velocity is $\tilde{\boldsymbol{v}}=(\tilde{u}, \tilde{v}, \tilde{w})$ and $\tilde{p}$ is a rescaled pressure: the actual excess pressure is $\rho_{0} \tilde{p}$, where $\rho_{0}$ is the constant background density. The buoyancy frequency $N$ is a positive constant and $\tilde{b}$ is the buoyancy. Henceforth, tildes indicate (real) quantities in the physical domain.

We remove the time dependence by Laplace transformation. Thus, we define, for example,

$$
p(x, y, z ; s)=\int_{0}^{\infty} \tilde{p}(x, y, z, t) \mathrm{e}^{-s t} \mathrm{~d} t .
$$

Assuming zero initial data, and eliminating $b$, we obtain

$$
s \boldsymbol{v}=-\operatorname{grad} p-\left(N^{2} / s\right) w \hat{\mathbf{z}}, \quad \operatorname{div} \boldsymbol{v}=0 .
$$

The first of these gives

$$
u=-\frac{1}{s} \frac{\partial p}{\partial x}, \quad v=-\frac{1}{s} \frac{\partial p}{\partial y} \quad \text { and } \quad w=-\frac{s}{N^{2}+s^{2}} \frac{\partial p}{\partial z} .
$$


Then, $\operatorname{div} \boldsymbol{v}=0$ gives

$$
\frac{\partial^{2} p}{\partial x^{2}}+\frac{\partial^{2} p}{\partial y^{2}}+\lambda^{2} \frac{\partial^{2} p}{\partial z^{2}}=0 \quad \text { with } \quad \lambda^{2}(s)=\frac{s^{2}}{N^{2}+s^{2}} .
$$

This is a partial differential equation for $p$. For time-harmonic problems (with a time dependence of $\mathrm{e}^{-\mathrm{i} \omega t}$ ), the same equations (for $p, u, v$ and $w$ ) are obtained but with $s=-\mathrm{i} \omega$; see $\S 5$.

\section{A horizontal plate in a stratified fluid}

We have a thin flat plate, $\Omega$, in the $x y$-plane. The rest of the $x y$-plane is denoted by $\Omega^{\prime}$. The motion is forced by oscillating the plate, with prescribed normal velocity on $\Omega, \widetilde{w_{\mathrm{f}}}$. Thus,

$$
\tilde{w}=\widetilde{w_{\mathrm{f}}}(x, y, t), \quad(x, y) \in \Omega, \quad t>0,
$$

which becomes, after Laplace transformation,

$$
w=-\frac{s}{N^{2}+s^{2}} \frac{\partial p}{\partial z}=w_{\mathrm{f}}(x, y ; s), \quad(x, y) \in \Omega .
$$

In fact, this holds on both sides of $\Omega$. It follows that $p$ must be an odd function of $z$, so the problem can be reduced to one in the half-space $z>0$. As we have split the problem into two half-space problems, we must also impose continuity of $p$ across $\Omega^{\prime}$.

Take the Fourier transform of (2.1) with respect to $x$ and $y$, with

$$
P(\xi, \eta, z)=\int_{-\infty}^{\infty} \int_{-\infty}^{\infty} p(x, y, z ; s) \mathrm{e}^{-\mathrm{i}(\xi x+\eta y)} \mathrm{d} x \mathrm{~d} y ;
$$

the inverse Fourier transform is

$$
p(x, y, z ; s)=\frac{1}{(2 \pi)^{2}} \int_{-\infty}^{\infty} \int_{-\infty}^{\infty} P(\xi, \eta, z) \mathrm{e}^{\mathrm{i}(\xi x+\eta y)} \mathrm{d} \xi \mathrm{d} \eta .
$$

(Henceforth, we write $\iint$ when the integration limits are as in (3.1) or (3.2).) Then, with $\kappa^{2}=\xi^{2}+\eta^{2}$, and writing $P^{\prime \prime}=\partial^{2} P / \partial z^{2}$, we obtain $P^{\prime \prime}=(\kappa / \lambda)^{2} P$. Hence,

$$
P(\xi, \eta, z)=C(\xi, \eta) \mathrm{e}^{(\kappa / \lambda) z}+D(\xi, \eta) \mathrm{e}^{-(\kappa / \lambda) z} \quad \text { for } z>0 .
$$

We require $p \rightarrow 0$ as $z \rightarrow \infty$, so we take $C=0$, assuming that $\operatorname{Re} \lambda \geq 0$.

Let us define the discontinuity in $p$ across $z=0$ by

$$
2 \delta(x, y) \equiv p(x, y, 0+; s)-p(x, y, 0-; s)=2 p(x, y, 0+; s) .
$$

Clearly, we must have $\delta=0$ on $\Omega^{\prime}$, so the Fourier transform of $\delta$ is

$$
\int_{\Omega} \delta(x, y) \mathrm{e}^{-\mathrm{i}(\xi x+\eta y)} \mathrm{d} x \mathrm{~d} y=D(\xi, \eta),
$$

using (3.3) and (3.4). Also, the Fourier transform of $w$ is given by

$$
W(\xi, \eta, z)=-s\left(N^{2}+s^{2}\right)^{-1} P^{\prime}=\kappa\left(N^{2}+s^{2}\right)^{-1 / 2} D(\xi, \eta) \mathrm{e}^{-(\kappa / \lambda) z} .
$$


Applying the boundary conditions gives

$$
\begin{array}{ll}
\frac{1}{(2 \pi)^{2}} \iint W(\xi, \eta, 0) \mathrm{e}^{\mathrm{i}(\xi x+\eta y)} \mathrm{d} \xi \mathrm{d} \eta=w_{\mathrm{f}}(x, y), & (x, y) \in \Omega, \\
\frac{1}{(2 \pi)^{2}} \iint P(\xi, \eta, 0) \mathrm{e}^{\mathrm{i}(\xi x+\eta y)} \mathrm{d} \xi \mathrm{d} \eta=0, & (x, y) \in \Omega^{\prime} .
\end{array}
$$

Substituting for $W(\xi, \eta, 0)$ and $P(\xi, \eta, 0)$ gives

$$
\begin{array}{ll}
\frac{1}{4 \pi^{2}} \iint \kappa D(\xi, \eta) \mathrm{e}^{\mathrm{i}(\xi x+\eta y)} \mathrm{d} \xi \mathrm{d} \eta=q(x, y), & (x, y) \in \Omega, \\
\iint D(\xi, \eta) \mathrm{e}^{\mathrm{i}(\xi x+\eta y)} \mathrm{d} \xi \mathrm{d} \eta=0, & (x, y) \in \Omega^{\prime},
\end{array}
$$

where

$$
q(x, y)=\left(N^{2}+s^{2}\right)^{1 / 2} w_{\mathrm{f}} .
$$

Equations (3.8) and (3.9) are a pair of dual integral equations for $D$. They are of a standard kind, so that various methods are available.

Another option is to substitute for $D$ in (3.8) using (3.5). This would lead to a hypersingular boundary integral equation over $\Omega$ for the discontinuity function, $\delta$.

To make analytical progress, we now suppose that $\Omega$ is circular.

\section{Circular disc}

\section{(a) Dual integral equations}

For a disc of radius $a$, use polar coordinates,

$$
x=r \cos \phi, \quad y=r \sin \phi, \quad \xi=\kappa \cos \beta, \quad \eta=\kappa \sin \beta,
$$

and suppose for simplicity that $\delta$ has a Fourier expansion in the form

$$
\delta=a \sum_{n=0}^{\infty} \epsilon_{n} d_{n}(r / a) \cos n \phi, \quad 0 \leq r<a,
$$

where $\epsilon_{0}=1$ and $\epsilon_{n}=2$ for $n \geq 1$. Using (3.5) and

$$
\mathrm{e}^{-\mathrm{i}(\xi x+\eta y)}=\mathrm{e}^{-\mathrm{i} \kappa r \cos (\phi-\beta)}=\sum_{m=0}^{\infty} \epsilon_{m}(-\mathrm{i})^{m} J_{m}(\kappa r) \cos m(\phi-\beta),
$$

we find that

$$
D=\sum_{n=0}^{\infty} \epsilon_{n} D_{n}(\kappa) \cos n \beta \quad \text { with } \quad D_{n}(\kappa)=2 \pi(-\mathrm{i})^{n} a \int_{0}^{a} d_{n}(r / a) J_{n}(\kappa r) r \mathrm{~d} r
$$

Then,

$$
\iint D \mathrm{e}^{\mathrm{i}(\xi x+\eta y)} \mathrm{d} \xi \mathrm{d} \eta=2 \pi \sum_{n=0}^{\infty} \epsilon_{n} \mathrm{i}^{n} \cos n \phi \int_{0}^{\infty} D_{n}(\kappa) J_{n}(\kappa r) \kappa \mathrm{d} \kappa,
$$


with a similar expansion for the integral in (3.8). Therefore, if $q$ has an expansion

$$
q=\sum_{n=0}^{\infty} \epsilon_{n} q_{n}(r / a) \cos n \phi, \quad 0 \leq r<a,
$$

the dual integral equations, (3.8) and (3.9), become, for $n=0,1,2, \ldots$,

$$
\begin{aligned}
\frac{\mathrm{i}^{n}}{2 \pi} \int_{0}^{\infty} \kappa^{2} D_{n}(\kappa) J_{n}(\kappa r) \mathrm{d} \kappa & =q_{n}(r / a), & & 0 \leq r<a, \\
\int_{0}^{\infty} \kappa D_{n}(\kappa) J_{n}(\kappa r) \mathrm{d} \kappa & =0, & & r>a .
\end{aligned}
$$

\section{(b) Expansion methods}

A standard method for solving integral equations (such as (4.6) and (4.7)) begins by expanding the unknown function in a series of basis functions. We should choose the expansion functions with two criteria in mind. First, $d_{n}(r / a)$ gives a radial component of $\delta$, and this quantity is known to have a square-root zero at the edge of the plate, $r=a$; this behaviour (which can be inferred from a local expansion near any point on the edge) should be incorporated in the expansion functions. Second, it would be useful if the integrals relating $d_{n}$ to $D_{n},(4.3)$, could be evaluated analytically for the chosen expansion functions. Of course, we may choose to expand $D_{n}$ directly (this is essentially Tranter's method (Tranter 1954, 1966)) but we prefer to begin with a quantity that has known physical properties.

Define a function $\Phi_{m}^{(n)}$ by

$$
\Phi_{m}^{(n)}(\rho)=\rho^{n} C_{2 m+1}^{n+1 / 2}\left(\sqrt{1-\rho^{2}}\right),
$$

where $C_{n}^{\lambda}$ is a Gegenbauer polynomial. As $\Phi_{m}^{(n)}(\rho)$ is of the form $\sqrt{1-\rho^{2}}$ multiplied by a polynomial in $\rho$, the square-root zero at the plate edge can be incorporated automatically. It can also be shown that $\Phi_{m}^{(n)}(\rho)$ is proportional to both

$$
P_{n+2 m+1}^{n}\left(\sqrt{1-\rho^{2}}\right) \text { and } \rho^{n} \sqrt{1-\rho^{2}} P_{m}^{(n, 1 / 2)}\left(1-2 \rho^{2}\right),
$$

where $P_{n}^{m}$ is an associated Legendre function and $P_{n}^{(\alpha, \beta)}$ is a Jacobi polynomial.

As Gegenbauer polynomials are orthogonal, so are $\Phi_{m}^{(n)}$. Perhaps more importantly, we have

$$
\int_{0}^{1} \Phi_{m}^{(n)}(x) J_{n}(\xi x) x \mathrm{~d} x=\frac{2 \Gamma(m+n+3 / 2)}{m ! \Gamma(n+1 / 2)} \frac{j_{n+2 m+1}(\xi)}{\xi},
$$

where $j_{n}(x)=\sqrt{\pi /(2 x)} J_{n+1 / 2}(x)$ is a spherical Bessel function. This formula is due to Tranter (1954, eqn (5); 1966, eqn (8.6)); see also (Krenk, 1979, eqn (72)).

We note in passing that the polynomials $r^{n} P_{m}^{(n, 0)}\left(1-2 r^{2}\right)$ have been advocated by Boyd $(2001, \S 18.5 .1)$ for use as radial basis functions in spectral methods for problems posed on a disc, $0 \leq r<1$. For us, the functions $\Phi_{m}^{(n)}$, defined by (4.8), are preferable because of the availability of Tranter's integral, (4.9), and because of the known behaviour at $r=1$. 


\section{(c) Solution of the dual integral equations}

Expand $\delta(r)$ as (4.2) with

$$
d_{n}(\rho)=\sum_{m=0}^{\infty} D_{m}^{n} \Phi_{m}^{(n)}(\rho)
$$

Then, from (4.3),

$$
\begin{aligned}
D_{n}(\kappa) & =2 \pi(-\mathrm{i})^{n} a^{3} \int_{0}^{1} d_{n}(\mu) J_{n}(\kappa a \mu) \mu \mathrm{d} \mu \\
& =4 \pi(-\mathrm{i})^{n} a^{3} \sum_{m=0}^{\infty} D_{m}^{n} \frac{\Gamma(m+n+3 / 2)}{\Gamma(n+1 / 2) m !} \frac{j_{n+2 m+1}(\kappa a)}{\kappa a},
\end{aligned}
$$

using (4.9). Substitution in (4.7) leads to the integral

$$
\int_{0}^{\infty} J_{n}(\kappa r) j_{n+2 m+1}(\kappa a) \mathrm{d} \kappa, \quad r>a
$$

this Weber-Schafheitlin integral (Watson, 1944, §13.4) vanishes, so that (4.7) is satisfied identically.

If we denote the left-hand side of (4.6) by $E_{n}(r / a)$, we have

$$
\begin{aligned}
E_{n}(\rho) & =\frac{\mathrm{i}^{n}}{2 \pi} \int_{0}^{\infty} \kappa^{2} D_{n}(\kappa) J_{n}(\kappa a \rho) \mathrm{d} \kappa \\
& =2 \sum_{m=0}^{\infty} D_{m}^{n} \frac{\Gamma(m+n+3 / 2)}{\Gamma(n+1 / 2) m !} L_{m}^{n}(\rho),
\end{aligned}
$$

where (Martin, 1986, p. 278)

$$
L_{m}^{n}(\rho)=\int_{0}^{\infty} \lambda J_{n}(\lambda \rho) j_{n+2 m+1}(\lambda) \mathrm{d} \lambda=\frac{\Gamma(n+1 / 2) \Gamma(m+3 / 2)}{m ! \sqrt{1-\rho^{2}}} \Phi_{m}^{(n)}(\rho),
$$

which is a polynomial in $\rho$. Hence, it follows that the right-hand side of (4.6) should have a similar polynomial expansion,

$$
q_{n}(\rho)=\sum_{m=0}^{\infty} Q_{m}^{n} \frac{\Phi_{m}^{(n)}(\rho)}{\sqrt{1-\rho^{2}}}
$$

and then

$$
Q_{m}^{n}=2 D_{m}^{n}(m !)^{-2} \Gamma(m+n+3 / 2) \Gamma(m+3 / 2) .
$$

This determines $D_{m}^{n}$ from $Q_{m}^{n}$; in general, these coefficients depend on $s$.

\section{(d) The pressure field}

The Laplace-transformed pressure is given by (3.2) and (3.3) (with $C=0$ ). If we expand $D$ as (4.3), we obtain

$$
p(x, y, z ; s)=\frac{1}{4 \pi^{2}} \sum_{n=0}^{\infty} \epsilon_{n} \iint D_{n}(\kappa ; s) \mathrm{e}^{-z \kappa / \lambda(s)} \mathrm{e}^{\mathrm{i}(\xi x+\eta y)} \cos n \beta \mathrm{d} \xi \mathrm{d} \eta .
$$


Substituting (4.11) and (4.13) gives

$$
p(x, y, z ; s)=\frac{a}{2 \pi} \sum_{n=0}^{\infty} \epsilon_{n}(-\mathrm{i})^{n} \sum_{m=0}^{\infty} \frac{m ! Q_{m}^{n} I_{n m}}{\Gamma(n+1 / 2) \Gamma(m+3 / 2)},
$$

where

$$
I_{n m}(s)=a^{2} \iint \frac{j_{n+2 m+1}(\kappa a)}{\kappa a} \mathrm{e}^{\mathrm{i}(\xi x+\eta y)-z \kappa / \lambda(s)} \cos n \beta \mathrm{d} \xi \mathrm{d} \eta, \quad z \geq 0 .
$$

To conclude, we invert, using

$$
\tilde{p}(x, y, z, t)=\frac{1}{2 \pi \mathrm{i}} \int_{c-\mathrm{i} \infty}^{c+\mathrm{i} \infty} \mathrm{e}^{s t} p(x, y, z ; s) \mathrm{d} s .
$$

As usual, $p$ is supposed to be analytic to the right of $\operatorname{Re} s=c>0$, ensuring that $p \equiv 0$ for $t<0$. From (2.1), we have

$$
\lambda^{-1}(s)=s^{-1}\left(N^{2}+s^{2}\right)^{1 / 2},
$$

with branch points at $s= \pm \mathrm{i} N$. We require that $\operatorname{Re} \lambda \geq 0$ for $\operatorname{Re} s \geq 0$ and we take the cuts to the left, parallel to the real $s$-axis. In addition, for timeharmonic forcing at a frequency $\omega<N$, the quantities $Q_{m}^{n}(s)$ will have poles at $s= \pm \mathrm{i} \omega$ (see (4.22) below). Then, moving the inversion contour to the left gives residue contributions from the poles and branch line integrals; the latter decay algebraically, they give the transient behaviour, and they are henceforth ignored as we are interested in the ultimate time-harmonic behaviour.

For the residue contributions, we note that

$$
\lambda^{-1}( \pm \mathrm{i} \omega)=\mp \mathrm{i} \tan \theta_{\mathrm{c}}, \quad 0<\omega=N \cos \theta_{\mathrm{c}}<N,
$$

and

$$
I_{n m}( \pm \mathrm{i} \omega)=I_{n m}^{ \pm}
$$

where $\omega=N \cos \theta_{\mathrm{c}}$ defines $\theta_{\mathrm{c}}, 0<\theta_{\mathrm{c}}<\pi / 2$, and

$$
I_{n m}^{ \pm}=a^{2} \iint \frac{j_{n+2 m+1}(\kappa a)}{\kappa a} \mathrm{e}^{\mathrm{i}(\xi x+\eta y) \pm \mathrm{i} \kappa z \tan \theta_{c}} \cos n \beta \mathrm{d} \xi \mathrm{d} \eta, \quad z \geq 0 .
$$

The substitutions $\xi \rightarrow-\xi$ and $\eta \rightarrow-\eta$ imply that $\beta \rightarrow \beta+\pi$ whence

$$
I_{n m}^{-}=(-1)^{n} \overline{I_{n m}^{+}},
$$

where the overbar denotes complex conjugation. Further discussion of the integrals $I_{n m}^{ \pm}$and their far-field behaviour can be found in $\S 7$.

To be more specific, suppose that

$$
\widetilde{w_{\mathrm{f}}}(x, y, t)=w_{\mathrm{p}}(x, y) \cos \omega t \quad \text { giving } \quad w_{\mathrm{f}}(x, y ; s)=s\left(s^{2}+\omega^{2}\right)^{-1} w_{\mathrm{p}}(x, y),
$$

where $w_{\mathrm{p}}$ does not depend on the transform variable, $s$. Then, from (3.10),

$$
q(x, y ; s)=s\left(s^{2}+N^{2}\right)^{1 / 2}\left(s^{2}+\omega^{2}\right)^{-1} w_{\mathrm{p}}(x, y) .
$$


So, if we expand the given function $w_{\mathrm{p}}$ as (cf. (4.5) and (4.12))

$$
w_{\mathrm{p}}(x, y)=\sum_{n=0}^{\infty} \epsilon_{n} \cos n \phi \sum_{m=0}^{\infty} U_{m}^{n} \frac{\Phi_{m}^{(n)}(r / a)}{\sqrt{1-(r / a)^{2}}}, \quad 0 \leq r<a,
$$

with (real) constants $U_{m}^{n}$, then

$$
Q_{m}^{n}(s)=s\left(s^{2}+N^{2}\right)^{1 / 2}\left(s^{2}+\omega^{2}\right)^{-1} U_{m}^{n} .
$$

Hence, (4.14) and (4.16) give

$$
\mathrm{e}^{s t} p(x, y, z ; s)=\frac{a}{2 \pi} \sum_{n=0}^{\infty} \epsilon_{n} \sum_{m=0}^{\infty} \frac{m ! U_{m}^{n} \mathcal{R}(s)}{\Gamma(n+1 / 2) \Gamma(m+3 / 2)},
$$

where $\mathcal{R}(s)=s^{2}\left(s^{2}+\omega^{2}\right)^{-1} \lambda^{-1}(s)(-\mathrm{i})^{n} I_{n m}(s) \mathrm{e}^{s t}$. Using (4.17), (4.18) and (4.20), the sum of the residues of $\mathcal{R}(s)$ at $s= \pm \mathrm{i} \omega$ is found to be

$$
(-\mathrm{i})^{n}\left\{I_{n m}^{+} \mathrm{e}^{\mathrm{i} \omega t}+I_{n m}^{-} \mathrm{e}^{-\mathrm{i} \omega t}\right\}(N / 2) \sin \theta_{\mathrm{c}}=\operatorname{Re}\left\{(-\mathrm{i})^{n} I_{n m}^{-} \mathrm{e}^{-\mathrm{i} \omega t}\right\} N \sin \theta_{\mathrm{c}} .
$$

Hence, inverting gives

$$
\tilde{p}(x, y, z, t)=\operatorname{Re}\left\{p(x, y, z) \mathrm{e}^{-\mathrm{i} \omega t}\right\}+\text { transients },
$$

where

$$
p(x, y, z)=\frac{a N}{2 \pi} \sin \theta_{\mathrm{c}} \sum_{n=0}^{\infty} \epsilon_{n}(-\mathrm{i})^{n} \sum_{m=0}^{\infty} \frac{m ! U_{m}^{n} I_{n m}^{-}}{\Gamma(n+1 / 2) \Gamma(m+3 / 2)} .
$$

Before considering specific forcings and their consequences, we examine the associated time-harmonic problem instead of solving an initial-value problem.

\section{The time-harmonic disc problem}

The pressure is supposed to have the form $\operatorname{Re}\left\{p(x, y, z) \mathrm{e}^{-\mathrm{i} \omega t}\right\}$, where $p$ satisfies

$$
\frac{\partial^{2} p}{\partial x^{2}}+\frac{\partial^{2} p}{\partial y^{2}}-\gamma^{2} \frac{\partial^{2} p}{\partial z^{2}}=0 \quad \text { with } \quad \gamma^{2}=\frac{\omega^{2}}{N^{2}-\omega^{2}}=\cot ^{2} \theta_{c} .
$$

As in $\S 3$, we consider $z>0$, with the plane $z=0$ partitioned into $\Omega$ (the plate) and $\Omega^{\prime}$, with $p=0$ on $\Omega^{\prime}$ and prescribed normal velocity on $\Omega$,

$$
w=\frac{\mathrm{i} \gamma^{2}}{\omega} \frac{\partial p}{\partial z}=w_{\mathrm{p}}(x, y), \quad(x, y) \in \Omega
$$

Introduce Fourier transforms,

$$
\begin{aligned}
& P(\xi, \eta, z)=\iint p(x, y, z) \mathrm{e}^{-\mathrm{i}(\xi x+\eta y)} \mathrm{d} x \mathrm{~d} y \\
& p(x, y, z)=\frac{1}{(2 \pi)^{2}} \iint P(\xi, \eta, z) \mathrm{e}^{\mathrm{i}(\xi x+\eta y)} \mathrm{d} \xi \mathrm{d} \eta .
\end{aligned}
$$


Then, with $\kappa^{2}=\xi^{2}+\eta^{2}$, the Fourier transform of $(5.1)$ is $P^{\prime \prime}=-(\kappa / \gamma)^{2} P$. Hence,

$$
P(\xi, \eta, z)=C(\xi, \eta) \mathrm{e}^{\mathrm{i}(\kappa / \gamma) z}+D(\xi, \eta) \mathrm{e}^{-\mathrm{i}(\kappa / \gamma) z} \text { for } z>0 .
$$

Equations (5.3) and (5.4) can be interpreted as giving a plane-wave decomposition of $p$. Thus, (5.1) has solutions in the form

$$
p(x, y, z)=\exp \left\{\mathrm{i}\left(k_{1} x+k_{2} y+k_{3} z\right)\right\} \quad \text { if } \quad \omega^{2}=N^{2}\left(k_{1}^{2}+k_{2}^{2}\right) / k^{2},
$$

where $k^{2}=k_{1}^{2}+k_{2}^{2}+k_{3}^{2}$. The corresponding group velocity, defined by $\boldsymbol{c}_{\mathrm{g}}=$ $\left(\partial \omega / \partial k_{1}, \partial \omega / \partial k_{2}, \partial \omega / \partial k_{3}\right)$, is given by

$$
\omega \boldsymbol{c}_{\mathrm{g}}=(N / k)^{2}\left(k_{1} \sin ^{2} \theta_{\mathrm{c}}, k_{2} \sin ^{2} \theta_{\mathrm{c}},-k_{3} \cos ^{2} \theta_{\mathrm{c}}\right) .
$$

So, in the half-space $z>0$, we may argue that the group velocity should be upwards, away from the disc, implying that we should take $k_{3}<0$, that is, we should take $C=0$ in (5.4). Thus, in effect, we have just applied a radiation condition. This idea has been used by Bell (1975), Nycander (2006) and others.

We now proceed as in $\S 3$. We define the discontinuity in $p$ across $z=0, \delta$, by (3.4); its Fourier transform, $D$, is given by (3.5). The Fourier transform of $w$ is given by

$$
W(\xi, \eta, z)=\mathrm{i}\left(\gamma^{2} / \omega\right) P^{\prime}=(\gamma \kappa / \omega) D(\xi, \eta) \mathrm{e}^{-\mathrm{i}(\kappa / \gamma) z} .
$$

Applying the boundary conditions gives (3.6) and (3.7), involving $W(\xi, \eta, 0)=$ $(\gamma / \omega) \kappa D$ and $P(\xi, \eta, 0)=D$. Hence, we obtain the dual integral equations, (3.8) and (3.9), for $D$, in which $q=(\omega / \gamma) w_{\mathrm{p}}=N w_{\mathrm{p}} \sin \theta_{\mathrm{c}}$.

For a circular disc, we expand $\delta$ and $w_{\mathrm{p}}$ using (4.2), (4.10) and (4.21), with known coefficients $U_{m}^{n}$ and unknown coefficients $D_{m}^{n}$. We have $Q_{m}^{n}=N U_{m}^{n} \sin \theta_{\mathrm{c}}$, with $Q_{m}^{n}$ related to $D_{m}^{n}$ by $(4.13)$ :

$$
N U_{m}^{n} \sin \theta_{\mathrm{c}}=2 D_{m}^{n}(m !)^{-2} \Gamma(m+n+3 / 2) \Gamma(m+3 / 2) .
$$

Then, we compute the pressure, using (5.3), (5.4) with $C=0,(4.3),(4.11)$ and (5.5): we obtain precisely the formula (4.24).

Summarising, we solved the time-harmonic problem with the radiation condition of upward-directed group velocity for each constituent plane wave. This led to exactly the same pressure field that we obtained by solving an initial-value problem, enforcing causality and ignoring transient effects.

Henceforth, we focus on the time-harmonic problem.

\section{Some examples}

We consider three simple examples. Two are axisymmetric, including a heaving rigid disc. The third is a rigid disc oscillating about a diameter. For each example, we solve the problem. Later, we shall calculate the far-field radiated field within the conical wave beams.

\section{(a) The heaving disc}

This is the simplest problem. It has been solved by Sarma \& Krishna (1972) and by Lai \& Lee (1981). We suppose that $w_{\mathrm{p}}=\mathcal{U}_{0}$, a given constant, so that we 
are only concerned with $n=0$. As $C_{1}^{\lambda}(x)=2 \lambda x, \Phi_{0}^{(0)}(\rho)=\sqrt{1-\rho^{2}}$ and so (4.21) gives $U_{0}^{0}=\mathcal{U}_{0}$, all other $U_{m}^{n}$ being zero.

The pressure is given by (4.24) as

$$
p(x, y, z)=\left(a \mathcal{U}_{0} / \pi^{2}\right) N \sin \theta_{\mathrm{c}} I_{00}^{-},
$$

with $I_{00}^{-}$defined by (4.19). The pressure discontinuity across the disc is $2 \rho_{0} \delta$, where

$$
\delta=a d_{0}(r / a)=D_{0}^{0} \sqrt{a^{2}-r^{2}}
$$

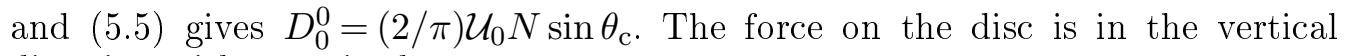
direction with magnitude

$$
\int_{\Omega} 2 \rho_{0} \delta \mathrm{d} A=4 \pi \rho_{0} \int_{0}^{a} \delta r \mathrm{~d} r=\frac{8}{3} \omega \mathcal{U}_{0} \rho_{0} a^{3} \tan \theta_{c}
$$

which agrees with (Lai \& Lee, 1981, eqn (47)).

\section{(b) Another axisymmetric problem}

As $C_{3}^{1 / 2}(x)=\frac{1}{2} x\left(5 x^{2}-3\right)$, we have $\Phi_{1}^{(0)}(\rho)=\frac{1}{2}\left(2-5 \rho^{2}\right) \sqrt{1-\rho^{2}}$. Therefore, from $(4.21)$,

$$
w_{\mathrm{p}}=\frac{1}{2} \mathcal{U}_{1}\left(2-5[r / a]^{2}\right) \text { gives } \quad U_{1}^{0}=\mathcal{U}_{1},
$$

all other $U_{m}^{n}$ being zero; here, $\mathcal{U}_{1}$ is a constant. The pressure is

$$
p(x, y, z)=(2 / 3)\left(a / \pi^{2}\right) \mathcal{U}_{1} N \sin \theta_{\mathrm{c}} I_{01}^{-} .
$$

The force on the disc is readily calculated.

\section{(c) The rolling disc}

We consider a rigid disc oscillating about a diameter, taken as the $y$-axis. For this 'rolling' motion, we have $w_{\mathrm{p}}=\mathcal{V}_{0} x / a=\mathcal{V}_{0}(r / a) \cos \phi$, where $\mathcal{V}_{0}$ is a given constant. Thus, $n=1$ and, as $\Phi_{0}^{(1)}(\rho)=3 \rho \sqrt{1-\rho^{2}}, U_{0}^{1}=\frac{1}{6} \mathcal{V}_{0}$, all other $U_{m}^{n}$ being zero. The pressure is

$$
p(x, y, z)=-\mathrm{i}(2 / 3)\left(a / \pi^{2}\right) \mathcal{V}_{0} N \sin \theta_{\mathrm{c}} I_{10}^{-}
$$

and the pressure discontinuity is

$$
\delta=2 a d_{1}(r / a) \cos \phi=6 D_{0}^{1}(r / a) \sqrt{a^{2}-r^{2}} \cos \phi,
$$

where $D_{0}^{1}=\frac{2}{9}(N / \pi) \mathcal{V}_{0} \sin \theta_{c}$. Thus, there is no net force on the disc but there is a moment about the $y$-axis with magnitude

$$
\int_{\Omega} 2 \rho_{0} \delta r \cos \phi \mathrm{d} A=\frac{12 \pi \rho_{0}}{a} D_{0}^{1} \int_{0}^{a} r^{3} \sqrt{a^{2}-r^{2}} \mathrm{~d} r=\frac{16}{45} \mathcal{V}_{0} \rho_{0} a^{4} N \sin \theta_{\mathrm{c}} .
$$

We are unaware of previous results on rolling discs. 


\section{The far field}

In the far field, $R \equiv \sqrt{r^{2}+z^{2}}$ is large. However, we are expecting characteristic conical wave beams, so it is useful to introduce conical polar coordinates, $\sigma$ and $\zeta$, defined by

$$
\sigma=r \cos \theta_{\mathrm{c}}-z \sin \theta_{\mathrm{c}} \quad \text { and } \quad \zeta=r \sin \theta_{\mathrm{c}}+z \cos \theta_{\mathrm{c}}
$$

equivalently, $\quad r=\sigma \cos \theta_{\mathrm{c}}+\zeta \sin \theta_{\mathrm{c}}$ and $z=-\sigma \sin \theta_{\mathrm{c}}+\zeta \cos \theta_{\mathrm{c}}$. There are two characteristic (double) cones that intersect the edge of the disc at $r=a$. Restricting to $z \geq 0, r \geq 0$, the lower cone is $z=(r-a) \cot \theta_{\mathrm{c}}$ with $r \geq a$, and the upper cone is $z=(r+a) \cot \theta_{\mathrm{c}}$. We expect to find energy propagation between these two cones, away from the disc. The lower cone corresponds to $\sigma=a \cos \theta_{\mathrm{c}}$ and the upper cone corresponds to $\sigma=-a \cos \theta_{c}$; the width of the beam is $2 b$ with $b=a \cos \theta_{\mathrm{c}}$.

\section{(a) The far-field pressure}

The pressure is given by (4.24) in terms of the integral $I_{n m}^{-}$, defined by (4.19). As $I_{n m}^{-}$is an even function of $y$, we can assume that $y \geq 0$.

To start, we use (4.1) in (4.19), with $0 \leq \phi \leq \pi$, giving

$$
I_{n m}^{-}=a \int_{0}^{\infty} \int_{0}^{2 \pi} j_{n+2 m+1}(\kappa a) \mathrm{e}^{\mathrm{i} \kappa r \cos (\beta-\phi)} \mathrm{e}^{-\mathrm{i} \kappa z \tan \theta_{c}} \cos n \beta \mathrm{d} \beta \mathrm{d} \kappa, \quad z \geq 0 .
$$

The integration with respect to $\beta$ can be evaluated but it turns out to be more convenient to retain the double integral.

In the far field, $\zeta \rightarrow \infty$ but $\sigma$ is finite; note that $\zeta \sim R$ in this limit. Then, (7.2) becomes

$$
I_{n m}^{-}=\int_{0}^{\infty} \int_{0}^{2 \pi} g(\kappa, \beta) \mathrm{e}^{\mathrm{i} \zeta f(\kappa, \beta)} \mathrm{d} \beta \mathrm{d} \kappa
$$

where

$$
\begin{aligned}
& g(\kappa, \beta)=a j_{n+2 m+1}(\kappa a) \mathrm{e}^{\mathrm{i} \kappa \sigma\left\{\cos (\beta-\phi) \cos \theta_{\mathrm{c}}+\tan \theta_{\mathrm{c}} \sin \theta_{\mathrm{c}}\right\}} \cos n \beta, \\
& f(\kappa, \beta)=\{\cos (\beta-\phi)-1\} \kappa \sin \theta_{\mathrm{c}} .
\end{aligned}
$$

The form of (7.3) suggests using the two-dimensional method of stationary phase. We have

$$
\frac{\partial f}{\partial \kappa}=\{\cos (\beta-\phi)-1\} \sin \theta_{c} \quad \text { and } \quad \frac{\partial f}{\partial \beta}=-\kappa \sin (\beta-\phi) \sin \theta_{c} .
$$

These both vanish when $\beta=\phi$, giving a line of stationary points. On this line, $f=0, f_{\kappa \kappa}=0, f_{\beta \beta}=-\kappa \sin \theta_{\mathrm{c}}$ and $g(\kappa, \phi)=a j_{n+2 m+1}(\kappa a) \mathrm{e}^{\mathrm{i} \kappa \sigma \sec \theta_{\mathrm{c}}} \cos n \phi$. Then, from (Wong, 2001, p. 454, Theorem 1), we obtain the estimate

$$
I_{n m}^{-} \sim b_{0} \zeta^{-1 / 2} \quad \text { with } \quad b_{0}=\sqrt{2 \pi} \mathrm{e}^{-\mathrm{i} \pi / 4} \int_{0}^{\infty} \frac{g(\kappa, \phi) \mathrm{d} \kappa}{\sqrt{\left|f_{\kappa \kappa}+f_{\beta \beta}\right|}} .
$$

Hence,

$$
I_{n m}^{-} \sim(a / \zeta)^{1 / 2} h_{n+2 m}(\sigma) \cos n \phi
$$


where

$$
h_{n}(\sigma)=\frac{\pi \mathrm{e}^{-\mathrm{i} \pi / 4}}{\sqrt{\sin \theta_{\mathrm{c}}}} \int_{0}^{\infty} J_{n+3 / 2}(\kappa a) \mathrm{e}^{\mathrm{i} \kappa \sigma \sec \theta_{\mathrm{c}}} \frac{\mathrm{d} \kappa}{\kappa} .
$$

This integral can be evaluated. From (Watson, 1944, p. 405, eqns (2) and (3)),

$$
\int_{0}^{\infty} J_{\mu}(\kappa a) \mathrm{e}^{ \pm \mathrm{i} \kappa c} \frac{\mathrm{d} \kappa}{\kappa}= \begin{cases}\mu^{-1} \exp ( \pm \mathrm{i} \mu \arcsin [c / a]), & 0 \leq c \leq a, \\ \mu^{-1}\left[c+\sqrt{c^{2}-a^{2}}\right]^{-\mu} a^{\mu} \mathrm{e}^{ \pm \mathrm{i} \pi \mu / 2}, & 0<a \leq c .\end{cases}
$$

Let $b=a \cos \theta_{c}$ and $\mu=n+\frac{3}{2}$. For $0 \leq \sigma \leq b$, we take the plus in the first of (7.5) with $c=\sigma \sec \theta_{\text {c }}$. For $-b \leq \sigma \leq 0$, we take the minus in the first of (7.5) with $c=-\sigma \sec \theta_{\mathrm{c}}$. Hence,

$$
h_{n}(\sigma)=\frac{2 \pi \mathrm{e}^{-\mathrm{i} \pi / 4}}{(2 n+3) \sqrt{\sin \theta_{\mathrm{c}}}} \mathrm{e}^{\mathrm{i}(2 n+3) \vartheta}, \quad|\sigma| \leq b=a \cos \theta_{\mathrm{c}},
$$

with

$$
\vartheta=\frac{1}{2} \arcsin (\sigma / b)
$$

The formula (7.6) shows the phase variation of $h_{n}(\sigma)$ across the wave beam. Outside the beam, we use the second of (7.5); for example,

$$
h_{n}(\sigma)=\frac{2 \pi \mathrm{i}^{n+1}}{(2 n+3) \sqrt{\sin \theta_{\mathrm{c}}}}\left\{(\sigma / b)+\sqrt{(\sigma / b)^{2}-1}\right\}^{-n-3 / 2}, \quad \sigma>b .
$$

Combining (7.4) and (7.6) gives, within the upper beam,

$$
I_{n m}^{-} \sim \frac{2 \pi \mathrm{e}^{-\mathrm{i} \pi / 4} \mathrm{e}^{\mathrm{i}(2 n+4 m+3) \vartheta}}{(2 n+4 m+3) \sqrt{\sin \theta_{\mathrm{c}}}} \sqrt{\frac{a}{\zeta}} \cos n \phi, \quad|\sigma| \leq b .
$$

Hence,

$$
p \sim \sqrt{\frac{a}{\zeta}} \sum_{n=0}^{\infty} \epsilon_{n} F_{n}(\sigma) \cos n \phi
$$

where

$$
F_{n}(\sigma)=a N \sqrt{\sin \theta_{c}} \frac{\mathrm{i}^{n} \mathrm{e}^{-\mathrm{i} \pi / 4}}{\Gamma(n+1 / 2)} \sum_{m=0}^{\infty} \frac{m ! U_{m}^{n} \mathrm{e}^{\mathrm{i}(2 n+4 m+3) \vartheta}}{\Gamma(m+3 / 2)(2 n+4 m+3)} .
$$

Notice that $p$ decays as $\zeta^{-1 / 2}$ for all forcings of the disc, whereas the behaviour across the beam (via $\vartheta$ ) depends on those values of $n$ and $m$ present in the forcing (see $(4.21))$.

We remark that integrals of the form (7.2) have been considered by Voisin (2003, eqn (4.21)). He obtained far-field estimates (see his (4.16)) but our method is much more direct. 


\section{(b) The far-field velocity}

In Cartesian coordinates, the (time-harmonic) velocity field is $\boldsymbol{v}=\left(u_{x}, u_{y}, w\right)$, where

$$
u_{x}=\frac{1}{\mathrm{i} \omega} \frac{\partial p}{\partial x}, \quad u_{y}=\frac{1}{\mathrm{i} \omega} \frac{\partial p}{\partial y}, \quad w=\frac{\mathrm{i} \gamma^{2}}{\omega} \frac{\partial p}{\partial z},
$$

$\gamma=C / S, C=\cos \theta_{\mathrm{c}}, S=\sin \theta_{\mathrm{c}}$ and $\omega=N C$. We use cylindrical polar coordinates $(r, \phi$ and $z)$ and conical polar coordinates $(\zeta, \sigma$ and $\phi)$, defined as follows (see $(7.1))$ :

$$
x=r \cos \phi, \quad y=r \sin \phi, \quad \zeta=r S+z C, \quad \sigma=r C-z S .
$$

Corresponding orthogonal unit vectors are $\hat{\boldsymbol{\zeta}}=(S \cos \phi, S \sin \phi, C), \quad \hat{\boldsymbol{\sigma}}=(C \cos \phi, C \sin \phi,-S), \quad \hat{\boldsymbol{\phi}}=(-\sin \phi, \cos \phi, 0)$.

Applications of the chain rule give

$$
\begin{aligned}
& \boldsymbol{v} \cdot \hat{\boldsymbol{\zeta}}=\frac{1}{\mathrm{i} N S} \frac{\partial p}{\partial \sigma}-\frac{\cos 2 \theta_{\mathrm{c}}}{\mathrm{i} \omega S^{2}} \frac{\partial p}{\partial \zeta} \\
& \boldsymbol{v} \cdot \hat{\boldsymbol{\sigma}}=\frac{1}{\mathrm{i} N S} \frac{\partial p}{\partial \zeta}, \quad \boldsymbol{v} \cdot \hat{\boldsymbol{\phi}}=\frac{1}{\mathrm{i} \omega(\zeta S+\sigma C)} \frac{\partial p}{\partial \phi} .
\end{aligned}
$$

Within the conical wave beams, $\zeta$ is large and $p$ decays as $\zeta^{-1 / 2}$ (see (7.7)), so that

$$
\boldsymbol{v} \sim v \hat{\boldsymbol{\zeta}} \quad \text { with } \quad v=(\mathrm{i} N S)^{-1} \partial p / \partial \sigma .
$$

Thus, using (7.7),

$$
v \sim \sqrt{\frac{a}{\zeta}} \sum_{n=0}^{\infty} \epsilon_{n} G_{n}(\sigma) \cos n \phi
$$

where $G_{n}(\sigma)=(\mathrm{i} N S)^{-1} F_{n}^{\prime}(\sigma)$. As $\vartheta=\frac{1}{2} \arcsin (\sigma / b), d \vartheta / d \sigma=\frac{1}{2}\left(b^{2}-\sigma^{2}\right)^{-1 / 2}$ and

$$
G_{n}(\sigma)=\frac{a \mathrm{i}^{n} \mathrm{e}^{-\mathrm{i} \pi / 4}}{2 \sqrt{b^{2}-\sigma^{2}} \sqrt{\sin \theta_{\mathrm{c}}} \Gamma(n+1 / 2)} \sum_{m=0}^{\infty} \frac{m ! U_{m}^{n} \mathrm{e}^{\mathrm{i}(2 n+4 m+3) \vartheta}}{\Gamma(m+3 / 2)} .
$$

(c) The far-field energy transport

The time-averaged energy transport vector is (see, for example, (Voisin, 2003, eqn $(4.39))$ )

$$
\mathbf{I}=\left(\rho_{0} / 2\right) \operatorname{Re}\{p \overline{\boldsymbol{v}}\} \sim\left(\rho_{0} / 2\right) \operatorname{Re}\{p \overline{\boldsymbol{v}}\} \hat{\boldsymbol{\zeta}} \quad \text { as } \zeta \rightarrow \infty .
$$

Let us calculate the total energy flux along the upper conical wave beam,

$$
\mathcal{E} \equiv \iint \mathbf{I} \cdot \hat{\boldsymbol{\zeta}} \mathrm{d} S=\frac{\rho_{0}}{2} \zeta \sin \theta_{\mathrm{c}} \int_{-b}^{b} \int_{-\pi}^{\pi} \operatorname{Re}\{p \bar{v}\} \mathrm{d} \phi \mathrm{d} \sigma,
$$

using $\mathrm{d} S \sim \zeta \sin \theta_{\mathrm{c}} \mathrm{d} \phi \mathrm{d} \sigma$ (Voisin, 2003, eqn (4.40)). Substituting (7.7) and (7.9), and integrating over $\phi$ gives

$$
\mathcal{E}=\pi a \rho_{0} \sin \theta_{\mathrm{c}} \sum_{n=0}^{\infty} \epsilon_{n} \int_{-b}^{b} \operatorname{Re}\left\{F_{n}(\sigma) \overline{G_{n}(\sigma)}\right\} \mathrm{d} \sigma .
$$


Use of (7.8) and (7.10) gives

$$
F_{n} \overline{G_{n}}=\frac{a^{2} N}{2[\Gamma(n+1 / 2)]^{2} \sqrt{b^{2}-\sigma^{2}}} \sum_{m=0}^{\infty} \sum_{\ell=0}^{\infty} \frac{\ell ! m ! U_{m}^{n} \overline{U_{\ell}^{n}} \mathrm{e}^{4 \mathrm{i}(m-\ell) \vartheta}}{\Gamma(\ell+3 / 2) \Gamma(m+3 / 2)(2 n+4 m+3)} .
$$

The remaining integral has the form

$$
\int_{-b}^{b} \frac{\mathrm{e}^{4 \mathrm{i} m \vartheta} \mathrm{d} \sigma}{\sqrt{b^{2}-\sigma^{2}}}=\int_{-\pi / 2}^{\pi / 2} \mathrm{e}^{2 \mathrm{i} m \xi} \mathrm{d} \xi=\pi \delta_{m 0},
$$

where $\delta_{i j}$ is the Kronecker delta. Hence,

$$
\mathcal{E}=\frac{1}{2} \pi^{2} a^{3} \rho_{0} N \sin \theta_{\mathrm{c}} \sum_{n=0}^{\infty} \frac{\epsilon_{n}}{[\Gamma(n+1 / 2)]^{2}} \sum_{m=0}^{\infty} \frac{(m !)^{2}}{[\Gamma(m+3 / 2)]^{2}} \frac{\left|U_{m}^{n}\right|^{2}}{(2 n+4 m+3)} .
$$

Evidently, this is positive: the total energy flux is always outwards. However, the energy transport vector, I, need not point outwards for all $\sigma$ and for all $\phi$, as we show next by example.

\section{(d) Some examples}

Define $\mathcal{I}(\sigma, \phi)$ by

$$
\mathbf{I} \cdot \hat{\boldsymbol{\zeta}} \sim \frac{a \rho_{0}}{2 \zeta} \mathcal{I}(\sigma, \phi) \quad \text { as } \zeta \rightarrow \infty .
$$

We calculate $\mathcal{I}$ for several examples.

Axisymmetric forcings

Start with axisymmetric forcing $(n=0)$. Then, we have

$$
\mathcal{I}(\sigma)=\operatorname{Re}\left\{F_{0} \overline{G_{0}}\right\},
$$

where

$$
F_{0} \overline{G_{0}}=\frac{a^{2} N}{2 \pi \sqrt{b^{2}-\sigma^{2}}} \sum_{m=0}^{\infty} \sum_{\ell=0}^{\infty} \frac{\ell ! m ! U_{m}^{0} \overline{U_{\ell}^{0}} \mathrm{e}^{4 \mathrm{i}(m-\ell) \vartheta}}{\Gamma(\ell+3 / 2) \Gamma(m+3 / 2)(4 m+3)} .
$$

For a heaving rigid disc $(\S 6 \mathrm{a}), U_{m}^{0}=\mathcal{U}_{0} \delta_{m 0}$ and

$$
\mathcal{I}(\sigma)=\frac{2 a^{2} N\left|\mathcal{U}_{0}\right|^{2}}{3 \pi^{2} \sqrt{b^{2}-\sigma^{2}}} .
$$

Similarly, for the forcing described in $\S 6 \mathrm{~b}, U_{m}^{0}=\mathcal{U}_{1} \delta_{m 1}$ and

$$
\mathcal{I}(\sigma)=\frac{8 a^{2} N\left|\mathcal{U}_{1}\right|^{2}}{63 \pi^{2} \sqrt{b^{2}-\sigma^{2}}}
$$

For these two examples, $\mathcal{I}(\sigma)>0$ for $|\sigma|<b$. However, suppose we combine the two forcings, retaining both $U_{0}^{0}$ and $U_{1}^{0}$. Then,

$$
F_{0} \overline{G_{0}}=\frac{2 a^{2} N}{63 \pi^{2} \sqrt{b^{2}-\sigma^{2}}}\left(21\left|U_{0}^{0}\right|^{2}+4\left|U_{1}^{0}\right|^{2}+14 U_{0}^{0} \overline{U_{1}^{0}} \mathrm{e}^{-4 \mathrm{i} \vartheta}+6 U_{1}^{0} \overline{U_{0}^{0}} \mathrm{e}^{4 \mathrm{i} \vartheta}\right) .
$$


Suppose, further, that $U_{1}^{0}=\frac{5}{2} \alpha U_{0}^{0}$, where $\alpha$ is a real parameter. Then

$$
\mathcal{I}(\sigma)=\frac{2 a^{2} N\left|U_{0}^{0}\right|^{2}}{63 \pi^{2} \sqrt{b^{2}-\sigma^{2}}}\left(21+25 \alpha^{2}+50 \alpha \cos 4 \vartheta\right),
$$

where $4 \vartheta=2 \arcsin (\sigma / b)$ varies from $-\pi$ to $\pi$ as $\sigma$ varies from $-b$ to $b$, across the beam. We can easily choose $\alpha$ so that $\mathcal{I}(\sigma)$ is negative in part of the beam; a simple choice is $\alpha=1$.

\section{Non-axisymmetric forcings}

For a plate rolling about a diameter $(\S 6 \mathrm{c})$, we have $n=1, U_{m}^{1}=\frac{1}{6} \mathcal{V}_{0} \delta_{m 0}$ and

$$
\mathcal{I}(\sigma, \phi)=4 \operatorname{Re}\left\{F_{1} \overline{G_{1}}\right\} \cos ^{2} \phi=\frac{8 a^{2} N\left|\mathcal{V}_{0}\right|^{2}}{45 \pi^{2} \sqrt{b^{2}-\sigma^{2}}} \cos ^{2} \phi
$$

which is positive in all directions $(\phi)$ and across the beam $(|\sigma|<b)$.

Suppose, now, that we combine heaving and rolling; the non-zero coefficients are $U_{0}^{0}=\mathcal{U}_{0}$ and $U_{0}^{1}=\frac{1}{6} \mathcal{V}_{0}$. We have

$$
\begin{gathered}
\mathcal{I}(\sigma, \phi)=\operatorname{Re}\left\{\left(F_{0}+2 F_{1} \cos \phi\right)\left(\overline{G_{0}}+2 \overline{G_{1}} \cos \phi\right)\right\} \\
=\frac{2 a^{2} N}{45 \pi^{2} \sqrt{b^{2}-\sigma^{2}}}\left(15\left|\mathcal{U}_{0}\right|^{2}+4\left|\mathcal{V}_{0}\right|^{2} \cos ^{2} \phi\right. \\
\left.\quad+2 \operatorname{Re}\left\{3 \mathrm{i} \mathcal{V}_{0} \overline{\mathcal{U}_{0}} \mathrm{e}^{2 \mathrm{i} \vartheta}-5 \mathrm{i} \mathcal{U}_{0} \overline{\mathcal{V}_{0}} \mathrm{e}^{-2 \mathrm{i} \vartheta}\right\} \cos \phi\right) .
\end{gathered}
$$

If we suppose, further, that $\mathcal{V}_{0}=\beta \mathcal{U}_{0}$, where $\beta$ is a real constant, then

$$
\mathcal{I}(\sigma, \phi)=\frac{2 a^{2} N\left|\mathcal{U}_{0}\right|^{2}}{45 \pi^{2} \sqrt{b^{2}-\sigma^{2}}}\left(15+4 \beta^{2} \cos ^{2} \phi-16 \beta \sin 2 \vartheta \cos \phi\right) .
$$

The sign of this quantity varies with $\beta$ and with position within the beam (via $\phi$ and $\vartheta$ ).

\section{Discussion}

We have solved a class of problems for oscillating horizontal discs. As there is uncertainty about the radiation condition in the frequency domain, we began by solving an initial-value problem in the time domain, where causality can be imposed. We also solved a boundary-value problem in the frequency domain, using plane-wave (Fourier) decompositions with waves selected so as to have outgoing group velocity. Both methods led to the same solution (once transient effects are discarded in the time domain).

We used our time-harmonic solution to solve specific problems, such as those for heaving and rolling rigid discs. It is expected that similar problems for elliptical discs may also be solved (Martin 1986).

We showed how to calculate the far field, using asymptotic methods. In particular, we estimated the pressure and velocity within the conical wave beams, and we showed that the total energy flux is outwards. 


\section{References}

Appleby, J. C. \& Crighton, D. G. 1987 Internal gravity waves generated by oscillations of a sphere. J. Fluid Mech. 183, 439-450.

Baines, P. G. 1971 The reflexion of internal/inertial waves from bumpy surfaces. J. Fluid Mech. 46, 273-291.

Barcilon, V. \& Bleistein, N. 1969 Scattering of inertial waves in a rotating fluid. Studies in Appl. Math. 48, 91-104.

Bardakov, R. N., Vasil'ev, A. Yu. \& Chashechkin, Yu. D. 2007 Calculation and measurement of conical beams of three-dimensional periodic internal waves excited by a vertically oscillating piston. Fluid Dynamics 42, 612-626.

Bell, T. H. 1975 Lee waves in stratified flows with simple harmonic time dependence. J. Fluid Mech. 67, 705-722.

Boyd, J. P. 2001 Chebyshev and Fourier spectral methods, 2nd edn. New York, NY: Dover.

Chashechkin, Yu. D., Vasil'ev, A. Yu. \& Bardakov, R. N. 2004 Fine structure of beams of a three-dimensional periodic internal wave. Doklady Earth Sciences 397A, 816-819.

Davis, A. M. J. \& Llewellyn Smith, S. G. 2010 Tangential oscillations of a circular disk in a viscous stratified fluid. J. Fluid Mech. 656, 342-359.

Flynn, M. R., Onu, K. \& Sutherland, B. R. 2003 Internal wave excitation by a vertically oscillating sphere. J. Fluid Mech. 494, 65-93.

Gabov, S. A. \& Pletner, Y. D. 1988 The problem of the oscillations of a flat disc in a stratified fluid. USSR Computational Math. \& Math. Phys. 28, issue 1, 41-47.

Hendershott, M. C. 1969 Impulsively started oscillations in a rotating stratified fluid. J. Fluid Mech. 36, 513-527.

Krenk, S. 1979 A circular crack under asymmetric loads and some related integral equations. $J$. Appl. Mech. 46, 821-826.

Lai, R. Y. S. \& Lee, C.-M. 1981 Added mass of a spheroid oscillating in a linearly stratified fluid. Int. J. Engng. Sci. 19, 1411-1420.

LeBlond, P. H. \& Mysak, L. A. 1978 Waves in the ocean. Amsterdam: Elsevier.

Lighthill, J. 1978 Waves in fluids. Cambridge: Cambridge University Press.

Longuet-Higgins, M. S. 1964 On group velocity and energy flux in planetary wave motions. Deep-Sea Res. 11, 35-42.

Martin, P. A. 1986 Orthogonal polynomial solutions for pressurized elliptical cracks. Quart. J. Mech. Appl. Math. 39, 269-287.

Nycander, J. 2006 Tidal generation of internal waves from a periodic array of steep ridges. $J$. Fluid Mech. 567, 415-432.

Sarma, L. V. K. V. \& Krishna, D. V. 1972 Oscillation of axisymmetric bodies in a stratified fluid. Zastosowania Matematyki 13, 109-121.

Tranter, C. J. 1954 A further note on dual integral equations and an application to the diffraction of electromagnetic waves. Quart. J. Mech. Appl. Math. 7, 317-325.

Tranter, C. J. 1966 Integral transforms in mathematical physics, 3rd edn. London: Methuen.

Vallis, G. K. 2006 Atmospheric and oceanic fluid dynamics. Cambridge: Cambridge University Press.

Voisin, B. 1991 Internal wave generation in uniformly stratified fluids. Part 1. Green's functions and point sources. J. Fluid Mech., (1991) 439-480.

Voisin, B. 2003 Limit states of internal wave beams. J. Fluid Mech. 496, 243-293.

Voisin, B., Ermanyuk, E. V. \& Flór, J.-B. 2010 Internal wave generation by oscillation of a sphere, with application to internal tides. J. Fluid Mech. 666, 308-357.

Watson, G. N. 1944 Theory of Bessel functions, 2nd edn. Cambridge: Cambridge University Press.

Wong, R. 2001 Asymptotic approximations of integrals. Philadelphia, PA: SIAM. 